\title{
Development and validation of diagnostic triage criteria for liver disease from a minimum data set enabling the 'intelligent LFT' pathway for the automated assessment of deranged liver enzymes
}

\author{
Michael Hugh Miller, ${ }^{1}$ Andrew Fraser, $^{2}$ Gillian Leggett, ${ }^{2}$ Alastair \\ MacGilchrist, ${ }^{3}$ George Gibson, ${ }^{4}$ James Orr, ${ }^{4}$ Ewan H Forrest, ${ }^{4}$ Ellie Dow, ${ }^{5}$ \\ William Bartlett, ${ }^{5}$ Chirstopher Weatherburn, ${ }^{5}$ Axel Laurell, ${ }^{1}$ Kirsty Grant, ${ }^{1}$ \\ Kathryn Scott, ${ }^{6}$ Ronald Neville, ${ }^{5}$ John F Dillon ${ }^{1}$
}

${ }^{1}$ Gut Group, University of Dundee, Ninewells Hospital and Medical School, Dundee, UK ${ }^{2}$ NHS Grampian, Aberdeen Royal Infirmary, Aberdeen, UK ${ }^{3}$ NHS Lothian, Royal Infirmary of Edinburgh, Edinburgh, UK ${ }^{4} \mathrm{NHS}$ Greater Glasgow and Clyde, Glasgow Royal Infirmary, Glasgow, UK

${ }^{5}$ NHS Tayside, Ninewells Hospital and Medical School, Dundee, UK ${ }^{6}$ Department of

Gastroenterology, University of Dundee, Dundee, UK

\section{Correspondence to} Dr Michael Hugh Miller, Gut Group, University of Dundee, Ninewells Hospital and Medical School, Dundee DD1 9SY, UK; michael.miller1@nhs.net

Received 18 October 2017 Revised 22 December 2017

Accepted 14 January 2018 Published Online First

7 February 2018

\section{Check for updates}

To cite: Miller $\mathrm{MH}$,

Fraser A, Leggett G, et al.

Frontline Gastroenterology

2018;9:175-182.

\section{ABSTRACT}

Background Liver function tests (LFTs) are commonly abnormal; most patients with 'incidental' abnormal LFTs are not investigated appropriately and for those who are, current care pathways are geared to find an explanation for the abnormality by a lengthy process of investigation and exclusion, with costs to the patient and to the health service.

Objective To validate an intelligent automatable analysis tool (iLFT) for abnormal liver enzymes, which diagnoses common liver conditions, provides fibrosis stage and recommends management

Design A retrospective case note review from three tertiary referral liver centres, with application of the iLFT algorithm and comparison with the clinician's final opinion as gold standard. Results The iLFT algorithm in $91.3 \%$ of cases would have correctly recommended referral or management in primary care. In the majority of the rest of the cases, iLFT failed safe and recommended referral even when the final clinical diagnosis could have been managed in primary care. Diagnostic accuracy was achieved in $82.4 \%$ of cases, consistent with the fail-safe design of the algorithm. Two cases would have remained in primary care as per the algorithm outcome, however on clinical review had features of advanced fibrosis.

Conclusion iLFT analysis of abnormal liver enzymes offers a safe and robust method of risk stratifying patients to the most appropriate care pathway as well as providing reliable diagnostic information based on a single blood draw, without repeated contacts with health services. Offers the possibility of high quality investigation and diagnosis to all patients rather than a tiny minority.

\section{BACKGROUND}

There has been a dramatic increase in the number of liver function tests (LFTs) requested in both primary and secondary care. The majority of tests are requested in connection with exploring undifferentiated illness and monitoring non-hepatic long-term health conditions, particularly those related to cardiovascular risk factor reduction. LFTs are often the gateway to further invasive and/or expensive investigations. It can be unclear whether an initial abnormal LFT $^{1}$ might signify current or future liver disease, significant disease in other organs or may be a temporary phenomenon of little clinical relevance. However, what is clear is that in around $20 \%$ of initial LFTs, one or more of the enzyme levels are abnormal. ${ }^{1}$ The next stage of diagnostic tests such as ultrasound, although not 'invasive', draws many well people or those without liver disease into hospital hepatology investigation pathways while leaving many abnormal LFTs 
unexplained. Newer non-invasive approaches such as the 'ELF test' ${ }^{2}$ are being evaluated to help diagnose fibrotic liver disease but have yet to find their place in the diagnostic pathway. Guidelines for primary care have been published for evaluation of abnormal LFTs in asymptomatic patients but did not cover further testing nor take account of costs to the patient or the health service. ${ }^{3}$ Additionally, research shows that few of these recommendations are followed in accordance with best practice standards. Tapper et $a l^{4}$ assessed the cost-effectiveness of a directed approach to investigating elevated alanine transaminase (ALT) compared with a non-directed approach, whereby a full liver panel was performed on the first abnormal ALT detection. They found the non-directed approach to be more cost-effective and provide one additional diagnosis per 100 compared with the directed approach, at the expense however of a higher false-positive rate. Furthermore, when they modelled the effect of different population prevalences of non-alcoholic fatty liver disease (NAFLD), alcohol-related liver disease (ARLD) and drug-induced liver injury, they found that the directed approach became more cost-effective at prevalences above 53\%,51.1\% and 13\%, respectively, rates higher than the general population prevalence.

This increase in testing occurs in the context of the prevalence and mortality from chronic liver disease rising rapidly in the last few decades. In the UK, liver disease is the only major disease area with an increasing death rate in those under 65 years and is now the third most common cause of death in men of this age. The most common causes of abnormal LFTs leading to chronic liver disease are NAFLD, ${ }^{5} \mathrm{ARLD}^{6}$ and hepatitis $\mathrm{C}$ virus infection (HCV). ${ }^{7}$ These conditions are becoming more prevalent and pose a considerable burden on the health service. ${ }^{8}$ However, there are many other causes of abnormal LFTs including biliary disease, drug reactions, reactions to systemic illness and hepatic infections. Despite the increasing testing of LFTs, patients continue to present with undiagnosed end-stage liver disease, which may have been preventable by earlier diagnosis. These include: autoimmune hepatitis, which is responsive to steroids, hepatitis $\mathrm{C}$ infection, which can be cured in most patients by antiviral drugs, and alcohol misuse, where brief interventions may be effective. ${ }^{9}$ The LFT abnormalities may also be consequent on serious disease elsewhere requiring treatment, such as malignancy where its early detection may improve the prognosis.

The Birmingham and Lambeth Liver Evaluation Testing Strategies study found fewer than $5 \%$ of cases with abnormal liver enzymes had a specific liver condition, either chronic or acute and only $1.3 \%$ of cases had a serious liver condition. ${ }^{10}$ The abnormal liver function Investigations evaluation (ALFIE) study, a population-based study, found that abnormal liver enzymes can be detected in over $21 \%$ of the population, with only $1.14 \%$ developing liver disease over a medium-term follow-up. ${ }^{1}$ The majority of the remaining LFT abnormalities being attributable to hepatic steatosis of various aetiologies or hepatic reactions to systemic illness. The one caveat to these large studies is that they do not have the prolonged follow-up that is required to explore the natural history of chronic liver disease.

Interpretation of abnormal LFTs remains challenging, especially so in a primary care setting where comorbidity and high workload makes focusing on single condition clinical pathways unrealistic. The obesity epidemic and historically high rates of alcohol consumption are likely to result in an increase in the discovery of abnormal LFTs. ${ }^{11}$ The anticipated burden of work is such that it may be expected that primary care will be inundated with patients concerned about whether they have liver disease and secondary care services inundated with referrals. There is a pressing need to risk stratify individuals with abnormal LFTs, allowing safe and effective triage, thus optimising access and efficiency of secondary care services.

To date, diagnostic algorithms for LFTs have attempted to diagnose everyone, in cycles of repeated testing of the LFTs with progressive adding of further more complex tests. An alternative approach is not to try to diagnose everyone but to identify those patients who can be classified easily and triaged. To do this, we defined a minimum diagnostic criteria that allowed the diagnosis and prognosis of a liver disease and classification into three groups: (A) those with liver disease that was simple and at early stage that could be managed in general practice, for example, alcohol-related fatty liver disease (ARFLD). (B) Those with complex or advanced disease that require secondary care management or further diagnostic input from secondary care, for example, suspected autoimmune liver disease. (C) Those without a clear diagnosis, negative screens for liver disease who would be managed by the general practitioner (GP) initially reviewing available results in the patient specific-context and planning further management according as is the current standard pathway.

Such criteria could be incorporated into an automated blood sciences laboratory management system, which would allow input of the additional clinical information, automated triggering of the additional blood tests and staging calculations, if abnormal LFTs were detected on the original patient sample. Finally, the diagnostic information and automated interpretation could be returned to the GP, electronically removing many steps of visits and repeated testing for efficient management.

\footnotetext{
AIMS

1. to describe the process of development of triage criteria

2. to validate the criteria in a large real world cohort of patients.
} 


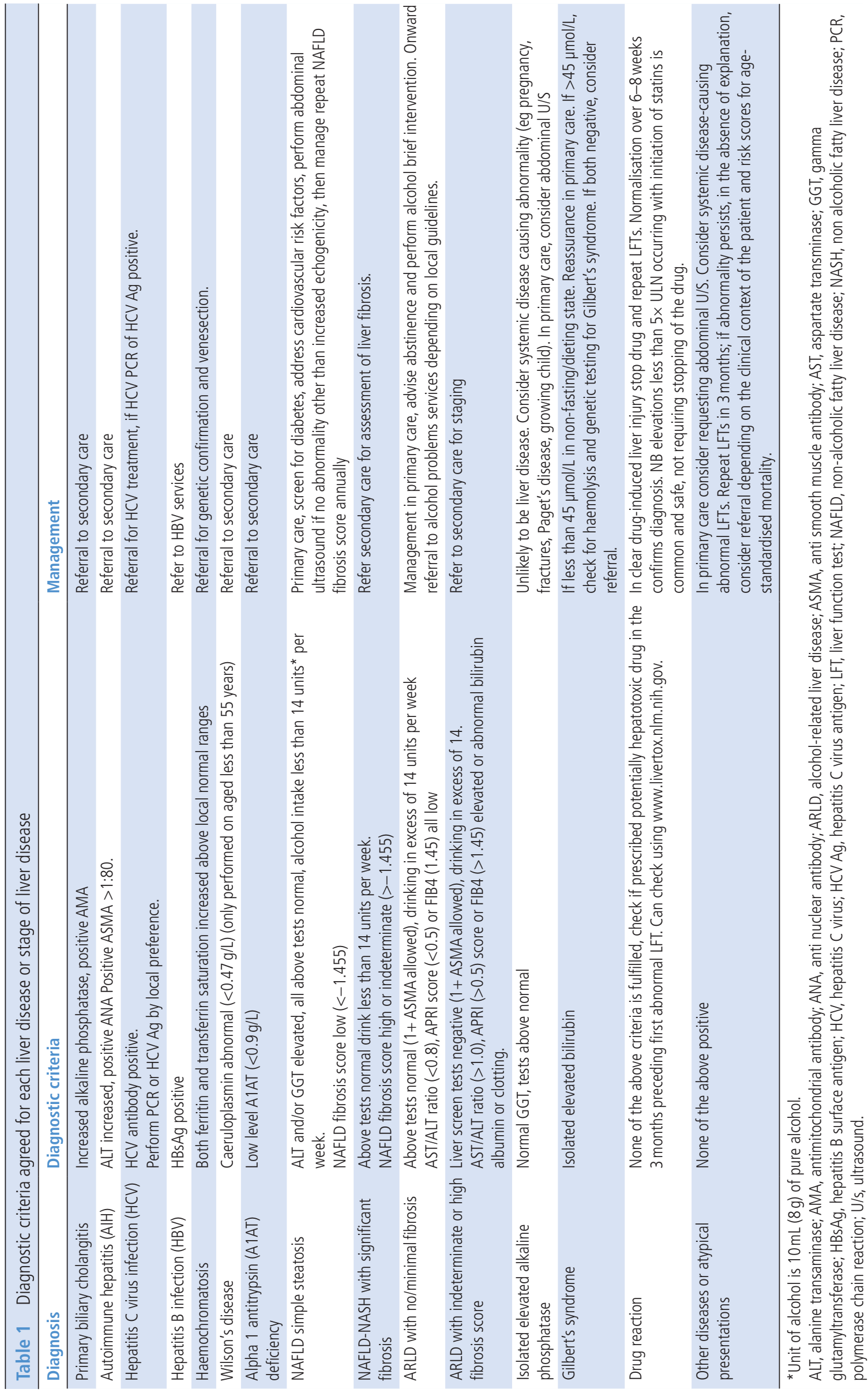


Table 2 Demographics and frequency of diagnoses for the entire cohort, as well as by site

\section{Entire}

cohort Dundee Aberdeen Glasgow

\begin{tabular}{lcclc}
\hline $\mathrm{n}$ & 323 & 190 & 41 & 92 \\
\hline Age (years) & 53.4 & 57 & 54 & 49.3 \\
\hline \% Female & $49.5 \%$ & $53 \%$ & $54 \%$ & $41 \%$ \\
\hline BMI & 29.9 & 30.3 & 29.5 & 29.4 \\
\hline Diagnosis & & & & \\
\hline PBC & 19 & 18 & 1 & 0 \\
\hline AlH & 19 & 17 & 2 & 0 \\
\hline HCV & 48 & 4 & $1^{*}$ & $43^{*}$ \\
\hline HBV & 9 & & $6^{*}$ & $3^{*}$ \\
\hline Haemochromatosis & 12 & 6 & 2 & 4 \\
\hline Wilson's disease & 0 & 0 & 0 & 0 \\
\hline A1AT & 4 & 4 & 0 & 0 \\
\hline NAFLD, no fibrosis & 78 & 60 & 8 & 10 \\
\hline NAFLD with fibrosis & 37 & 21 & 8 & 8 \\
\hline ARLD, no fibrosis & 21 & 16 & 2 & 3 \\
\hline ARLD with fibrosis & 24 & 8 & 1 & 15 \\
\hline Isolated elevated Alk & & & & \\
\hline Phos & 10 & 7 & 0 & 3 \\
\hline Gilbert's syndrome & 0 & 0 & 0 & 0 \\
\hline DILI & 8 & 8 & 0 & 0 \\
\hline Unclear & 34 & 21 & 10 & 3 \\
\hline On case of & & & \\
\hline
\end{tabular}

*One case of HBV/HCV coinfected.

A1AT, alpha 1 antitrypsin; AlH, autoimmune hepatitis; Alk Phos, alkaline phosphatse; ARLD, alcohol-related liver disease; BMI, body mass index; DILI, drug-induced liver injury; HBV, hepatitis B virus infection; $\mathrm{HCV}$, hepatitis C virus infection; NAFLD, non-alcoholic fatty liver disease; $\mathrm{PBC}$, primary biliary cholangitis.

\section{METHOD}

\section{Development of criteria}

An expert group of five hepatologists met on several occasions, tasked with using Delphi methodology. ${ }^{12}$ They were tasked with defining objective criteria to allow a confident diagnosis of common liver diseases based on a limited number of parameters. Specifically, the criteria were not required to diagnose everyone with a disease

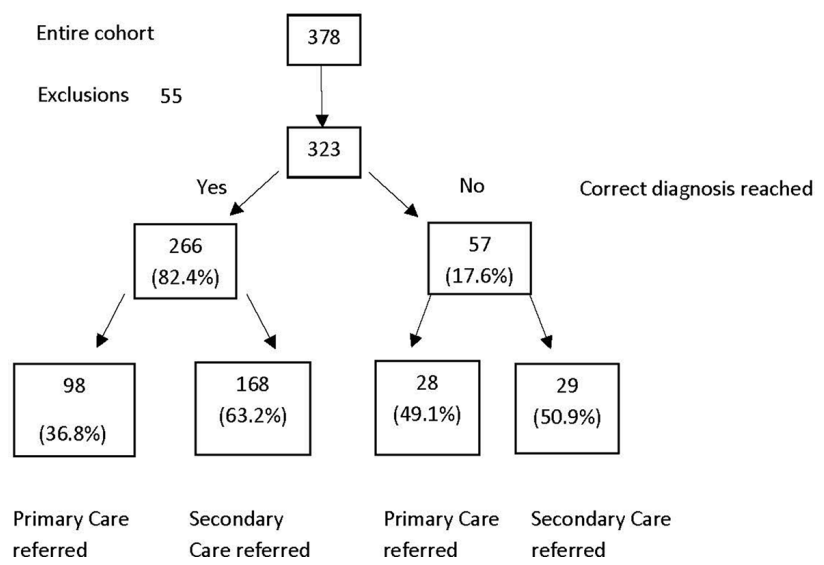

Figure 1 Diagnostic agreement between the automated process and the clinical diagnosis. and had to be based on a limited information set without clinical examination. Patients with overt jaundice were excluded, and it was also assumed that any derived diagnosis would be advisory and reviewed by a practitioner when the result of an abdominal ultrasound became available later.

The available information set was:

- patient-specific factors: age, sex, body mass index (BMI), features of the metabolic syndrome and alcohol intake

- the results of a liver disease aetiology screen: hepatitis serology, liver immunology, ferritin, alpha-1-antitrypsin and caeruloplasmin

- fibrosis staging: FIB4 and the NAFLD fibrosis score.

The criteria employed assessment of liver fibrosis by means of non-invasive fibrosis panels, in order to risk stratify cases. The NAFLD Fibrosis Score was used for cases where NAFLD was the suspected diagnosis. ${ }^{13}$ All cases scoring $>-1.455$ (ie, indeterminate cases as well as cases of F3/4) were recommended for referral. For all other liver conditions, both Fibrosis 4 (FIB4) ${ }^{14}$ and AST to Platelet Ratio Index (APRI) ${ }^{15}$ were used. If both or either suggested indeterminate or advanced fibrosis, referral was recommended.

The expert panel agreed on a list of common and or important liver diseases (see table 1); for each disease or disease stage, each panellist, using the available information, defined combinations that allowed confident diagnosis of the condition. These were collated, and all results were feed back to the experts, with conflicts of opinion and reasoning for them shared. Additionally, at this stage, mutual exclusivity was checked between the diagnostic criteria and overlaps feed back to the experts. The responses were collated and the criteria used on a consecutive series of referrals to a liver clinic for abnormal LFTs. The collated criteria and performance in the case note series was fed back to the experts, and consensus was achieved.

For some conditions, a clear and definitive diagnostic test exists (ie, HCV). For other conditions, a more detailed biochemical, virological and immunological panel, either positive or negative, is required before a diagnosis can be reached. In scenarios where there were unlikely diagnoses but different treatment options or prognosis, the criteria were designed to trigger a referral. Such scenarios are most common in patients with ARFLD or NAFLD with simple steatosis who have positive liver immunology, raising the rare but important possibility that they have autoimmune liver disease. In these cases, the criteria would allocate a possible diagnosis of autoimmune disease that would trigger referral to secondary care for expert assessment. This policy of diagnostic 'fail safe' was built into all the criteria. HCV, HBV and autoimmune liver conditions or NAFLD and ARFLD with indeterminate or elevated fibrosis staging were considered for referral to specialist services. NAFLD and ARFLD with low (F0-2) non-invasive fibrosis scores were recommended for management in primary care. The group where a robust diagnosis was not reached comprised mainly of cases of NAFLD who 
Table 3 Diagnostic agreement/disagreement for the various aetiologies encountered with clinical opinion at clinical review as gold standard

\begin{tabular}{|c|c|c|}
\hline & $\begin{array}{l}\text { Diagnostic } \\
\text { agreement }\end{array}$ & $\begin{array}{l}\text { Diagnostic } \\
\text { disagreement }\end{array}$ \\
\hline$n$ & 266 & 57 \\
\hline PBC & 17 & 2 \\
\hline AlH & 4 & 14 \\
\hline $\mathrm{HCV}$ & 46 & 0 \\
\hline HBV & 8 & 0 \\
\hline HFE & 14 & 1 \\
\hline Wilson's disease & 0 & 0 \\
\hline A1AT & 0 & 4 \\
\hline NAFLD, no fibrosis & 90 & 16 \\
\hline NAFLD with fibrosis & 10 & 3 \\
\hline ARLD, no fibrosis & 20 & 4 \\
\hline ARLD with fibrosis & 12 & 0 \\
\hline Isolated elevated ALP & 6 & 4 \\
\hline Gilbert's syndrome & 0 & 0 \\
\hline DILI & 11 & 0 \\
\hline Unclear & 28 & 9 \\
\hline
\end{tabular}

A1AT, alpha 1 antitrypsin; AlH, autoimmune hepatitis; ALP, alkaline phosphtase; ARLD, alcohol-related liver disease; DILI, drug-induced liver injury; HBV, hepatitis B virus infection; $H C V$, hepatitis C virus infection; HFE, hereditary haemochromatosis; NAFLD, non-alcoholic fatty liver disease; $\mathrm{PBC}$, primary biliary cholangitis.

do not have overt obesity or other features of the metabolic syndrome, those in whom history of alcohol excess was not initially discovered, unusual liver diseases and those with systemic illness and or a non-liver cause of elevation of an LFT analyte. These cases are highlighted for GP review in the same manner as is current practice but with a much reduced likelihood of parenchymal liver disease. All recommendations were provisional on the result of abdominal ultrasound scanning. Information sheets for GPs were produced for each of the liver conditions with brief descriptions of the condition, recommendations for the next investigation and management steps as well as the referral recommendation.

\section{Validation of criteria}

The criteria for diagnosis were validated against a retrospective case note validation study of consecutive new patient referrals with abnormal LFTs to the liver services at three tertiary referral centres. Patients with focal lesions, patients with known imaging demonstrating a dilated biliary tree, patients referred for management of a diagnosed liver disease and patients with jaundice were excluded. A researcher extracted data for the minimum diagnostic criteria and the final diagnosis and outcome of the referral, using the hospital liver clinicians' opinion as the gold standard for diagnosis. The criteria-based diagnosis and the referral recommendation were compared with the clinician's diagnosis. The accuracy of the pathway was assessed based on the number of correct diagnoses and referral decisions using the algorithm and against the final 'fail safe criteria' of all recommendations for primary care management. We examined in detail all cases of diagnostic disagreement, with particular focus on cases where the automated process suggested primary care management when specialist referral and review was warranted.

\section{RESULTS}

A total number of 378 cases were included in the validation cohort for the study. Fifty-five cases were found to be jaundiced (bilirubin $\geq 22$, range 22-117) and therefore removed from the analysis. Thus, 323 cases were included. Table 2 shows the demographics of cohort studied.

\section{Diagnostic agreement}

There was agreement in the diagnosis reached with the automated pathway in $82.4 \%$ of cases $(n=266)$. In all of these cases, the appropriate referral option would have been selected by the automated process. One hundred sixty-eight cases were allocated to the refer to secondary care group (52\% of the entire cohort) and 98 cases were allocated to the manage in GP group (30.3\% of the entire cohort).

\section{Diagnostic disagreement}

There was diagnostic disagreement between the automated pathway and the responsible clinician in $17.6 \%$ of the cases assessed. Of those 57 cases, 29 cases would have been referred to secondary care. Despite the incorrect diagnosis, 12 of those 29 cases required a secondary care opinion. The remaining 17 cases did not require a secondary care opinion.

The majority of cases had underlying alcohol related liver disease or NAFLD, with positive autoantibodies, an elevated ferritin level, elevated fibrosis scores or borderline alpha 1-antitrypsin levels. Thus the criteria performed as they were designed to do and failed safe, suggesting a possible diagnosis that would require secondary care opinion to investigate or exclude.

Figure 1 describes the cases of diagnostic agreement and disagreement, as well as the ultimate referral destination. Table 3 describes by disease aetiology where there was agreement and disagreement between the automated process and the clinical diagnosis.

\section{Referral recommendation}

The correct referral pathway was selected in 295 cases $(91.3 \%)$ of cases, regardless of the accuracy of the final diagnosis. There were 28 cases where the referral pathway was not appropriate $(8.6 \%)$. Figure 2 describes the referral recommendation accuracy, showing the number of patients referred to the correct destination (primary or secondary care).

Using the ultimate referral decision, rather than the actual diagnosis, the automated intervention has a sensitivity of $94.3 \%$ and a specificity of $86 \%$. 
Twenty-eight cases would have remained in primary care, 23 appropriately so despite an incorrect diagnosis. Five cases $(1.5 \%$ of the total cohort) would have remained in primary care but required a secondary care referral.

1. One case had an ultimate diagnosis of varicella-zoster-related enzyme derangement - the automated process recorded a diagnosis of ARLD with no evidence of significant fibrosis.

2. A case of acute hepatitis E virus (HEV) was misclassified as NAFLD with normal fibrosis scores. The protocol for NAFLD diagnosis uses an upper limit of $300 \mathrm{IU} / \mathrm{L}$ for NAFLD. At the time of referral, this patient had an ALT below 300 .

3. A case with features of the metabolic syndrome, an ALT of $91 \mathrm{U} / \mathrm{L}$ and a negative liver screen. The NAFLD score was -1.52 (low risk). The criteria suggested a diagnosis of NAFLD with no evidence of significant fibrosis. At clinical review, a Fibroscan was elevated, and the patient subsequently diagnosed with NASH-related cirrhosis.

4. A case of portal, splenic and superior mesenteric vein (SMV) thrombosis was classified as NAFLD with normal fibrosis scores.

5. A case of ARLD-related cirrhosis was classified as ARLD with normal fibrosis scores, as the FIB4 value was calculated at 1.31. However, on clinic review, the patient had evidence of portal hypertension.

Of the cases that were misallocated, two (the HEV and varicella-zoster virus) are self-limiting usually, and if the algorithm had been used on diagnosis bloods rather than clinic bloods when the transaminitis had resolved, they would have been correctly allocated. The portal vein thrombosis would have been picked up on the abdominal ultrasound examination that would still be part of the evaluation of these patients. The two patients with cirrhosis not detected by the fibrosis markers reflect the known fail rate for these tests and

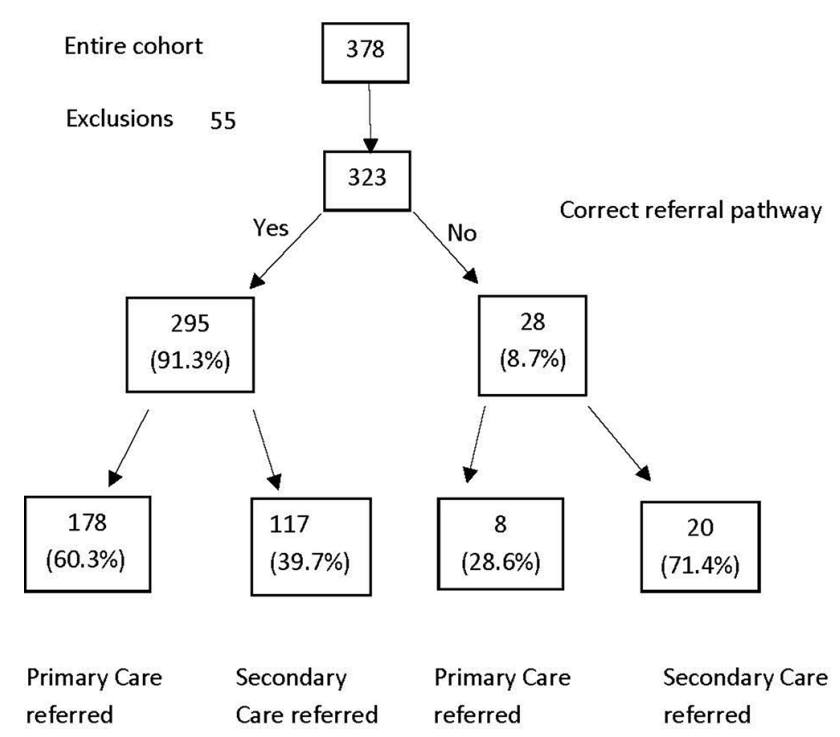

Figure 2 Referral pathway recommendations. need to be viewed in terms of missing many more patients if no tests were done.

\section{Potential impact}

We examined 323 cases in total, all of whom had been reviewed in secondary care. With the application of these criteria, 116 (35.9\%) could have been managed in primary care without the need for a specialist referral. The majority of these cases were either NAFLD or ARLD with low non-invasive fibrosis markers, These cases required lifestyle advice about diet and exercise or an alcohol brief intervention. This is already a population selected for referral so a higher proportion of primary care management would be expected in an unselected population. A proportion of those classed as requiring secondary care intervention was on the basis of indeterminate values from non-invasive fibrosis assessment methods. The use of advanced fibrosis markers such as the ELF panel or direct access to liver stiffness measurement could further reduce referral.

\section{DISCUSSION}

Only a fraction of individuals with abnormal liver enzymes detected in primary care go on to develop significant liver disease in medium-term follow-up. In some studies, this is as low as $1.4 \%-5 \%,{ }^{116}$ but despite this, as a result of the vast number of tests performed, this still represents a huge impact on public health. The intelligent use of healthcare resources described here could allow appropriate referral and triage of patients at greatest risk of significant liver disease while simultaneously providing additional support and information to primary care givers for those patients at less risk.

A problem with the assessment of abnormal LFTs is that for many patients the consideration of multiple factors by an expert in liver disease is required to arrive at a diagnosis. This has lent an 'air of mystique' to the diagnosis of liver disease. This study shows this need not be the case: many patients can be triaged simply and effectively at point of first presentation of abnormal LFTs. This decision support tool for GPs and the $20 \%$ of their patients who have abnormal LFTs tested for a variety of reasons ${ }^{1}$ could transform the management. Further work is needed to quantify just how significant an impact this could have on patients and primary and secondary care services.

The project described here arose from a working group convened by the Scottish government. The Liver Care Pathway Advisory Group focused on the problem of late presentation of liver disease with tragic consequences and the difficulties of spotting liver disease early among all the patients with abnormal LFTs. The project has used an extensive literature review and expert opinion to achieve professional consensus on minimum diagnostic criteria for liver diseases. The criteria define the liver diseases of interest and define 
the general practice role in each diagnostic group, that is, therapeutic management within primary care or referral for specialist management. In essence, for each liver disease, a set of highly specific diagnostic criteria have been identified based on a few simple clinical observations and blood test results. This allows diagnosis using a minimal number and values of diagnostic criteria that gives confidence that those identified do have the disease and accepts that others might have the disease but does not attempt to be all inclusive. These criteria allow patients to be allocated to one of three groups: those who have serious or complex disease that requires secondary care management, those who have less serious disease that can be managed within primary care and those in whom the diagnosis is not clear. This study investigated the utility of this approach to the diagnosis of liver disease compared with conventional services; clearly it has demonstrated that the algorithm functioned well in this unselected cohort of secondary care referrals.

The criteria and algorithm described in this study could streamline practice in relation to abnormal liver enzymes, reducing delays and associated costs to performing second-line investigations (a liver disease aetiology screen). We have shown the algorithm to be robust and risk averse, ensuring those with markers of alternative diseases or indeterminate fibrosis markers are still referred for secondary care opinion or investigation. If this process was automated, which it lends itself to, it could reduce contact time and episodes in general practice and reducing the burden of potential referrals to secondary care clinics.

The system makes extensive use of fibrosis scoring algorithms to stage patient's liver disease; these include the NAFLD fibrosis score, APRI and FIB4. The NAFLD Fibrosis Score (NAFLD FS) is widely used in secondary care settings, with many units using the score to risk stratify patients with NAFLD. ${ }^{13}$ Furthermore, a NAFLD FS of intermediate or high at presentation is associated with a significant risk of liver events and mortality with an HR of 7.7 and 34.2, respectively. ${ }^{13}$ In this project, NAFLD FS has been used to risk stratify patients with NAFLD. Those with low scores are recommended for primary care management, while those with indeterminate or high scores are recommended for referral for further staging tests such as liver stiffness measurement or biopsy. One potential weakness of this approach is the long-term follow-up of these patients. There is no evidence to support the serial measurement of the NAFLD FS in order to detect the development of fibrosis. Pragmatically, we advise the primary caregiver to prescribe lifestyle intervention measures and repeat the NAFLD FS on annual basis. For other aetiologies of liver disease, APRI ${ }^{15}$ and FIB $4^{14}$ are well-validated non-invasive fibrosis assessing tools. Serum-based non-invasive fibrosis detection algorithms have limitations. They perform well at detection of advanced disease but are not infallible and should be interpreted accordingly. Erring on the side of caution, we suggested the measurement of both for all liver conditions other than NAFLD. If either APRI or FIB4 did not suggest minimal fibrosis, referral was suggested. Within our study cohort, this occurred in fewer than five cases. This combined use of the two distinct tools is novel and pragmatic, with a fail-safe approach in mind. Abdominal ultrasound scan (AUSS) is useful in the detection of fatty infiltration of the liver as well as lesion detection (including gallstones) but has a limited role in the diagnosis of autoimmune and viral liver conditions; its role in this setting is an exclusionary one.

The overall accuracy of patient allocation to secondary care referral or primary care follow-up was very high. The diagnosis accuracy was less good, but this is an artificial effect reflecting the intention and design of the diagnostic criteria to fail safe. The majority of the apparent incorrect diagnosis from the criteria is due to either the possibility of autoimmune liver disease because of positive antismooth muscle antibodies in patients with ARLD or NAFLD or patients with elevated fibrosis scores requiring further secondary care investigation to define the degree of fibrosis. New more accurate blood tests for fibrosis, for instance ELF, are not routinely available to general practice, and fibroelastography is not currently available directly to primary care. Increased availability of either of these technologies could further reduce the need for referral.

These diagnostic criteria can be applied by clinicians to patients in the run of a clinical consultation, and they would be an aid to accurate and efficient diagnosis. However, they were designed to enable automated management. Technological developments within blood science laboratories have led to computerised test ordering and communications systems and the use of tracked analysers where patient samples are passed between analysers using microrail systems, which are computer controlled. This means that in real time, the system can change a sample's journey based on the preceding results for that sample. A new care pathway could be developed from the diagnostic criteria validated in this study and existing laboratory technology that flows as follows:

1. If a GP requests LFTs in one of the standard electronic requesting systems, a simple box opens, asks if they want to screen for liver disease if results are abnormal.

2. A positive response prompts the GP to enter data about patients' alcohol consumption and BMI.

3. In the laboratory, if such a sample is detected to have an abnormality, a cascade of additional aetiological tests will be performed and staging algorithms will be calculated.

4. The diagnostic criteria are used to give a diagnosis and management plan ranging from management in general practice to referral for secondary care.

5. In those where there is no definitive diagnosis, the GP is free to manage the patient as is standard practice now. 


\section{Significant of this study}

\section{What is already known on this topic}

- Currently, there is significant heterogeneity in the handling and analysis of deranged liver enzymes.

- This creates uncertainty, unnecessary investigations and delays in diagnosis.

- There is also an associated cost burden with the current system of liver enzyme assessment.

\section{What this study adds}

- This novel automated approach streamlines the analysis and subsequent investigation of deranged liver enzymes, providing a structured methodical approach.

- Employing non-invasive fibrosis scores allows safe identification of patients with more advanced liver disease.

- This structured automated approach demystifies liver enzyme derangement for the primary care provider.

\section{How might it impact on clinical practice in the} foreseeable future

- A structured and automated analysis of deranged liver enzymes streamlines patient investigations and referrals, with the potential impact of a higher, earlier rate of detection of liver disease. There is the potential for significant cost savings in the patient journey without compromise on patient safety.

6. All these data and information are passed back to the GP in a user-friendly format based within existing systems.

This system is now the subject of an ongoing clinical trial.

\section{Conclusions}

This study suggested that over $35.6 \%$ of referrals could have been managed in primary care without a specialist opinion. The majority of this cohort were patients with NAFLD or ARLD and low fibrosis scores. For these patients, we recommend primary care follow-up with either a lifestyle intervention programme or an alcohol brief intervention. Information sheets detailing this advice are automatically sent to the referring primary care provider. Such a reduction in referral to secondary care services has the potential to dramatically reduce the referral rate, allowing more appropriate use of a limited resource. Additionally, if the process was automated ensuring a higher rate of investigation of abnormal LFTs, there would be capacity in the system to cope with this quality improvement.

Contributors All authors contributed to the conception and data collection processes as well as manuscript editing. MHM coordinated the study and performed data analysis and manuscript preparation. JFD maintained study oversight.

Funding This research received no specific grant from any funding agency in the public, commercial or not-for-profit sectors.
Competing interests None declared.

Provenance and peer review Not commissioned; externally peer reviewed.

(C) Article author(s) (or their employer(s) unless otherwise stated in the text of the article) 2018. All rights reserved. No commercial use is permitted unless otherwise expressly granted.

\section{REFERENCES}

1 Donnan PT, McLernon D, Dillon JF, et al. Development of a decision support tool for primary care management of patients with abnormal liver function tests without clinically apparent liver disease: a record-linkage population cohort study and decision analysis (ALFIE). Health Technol Assess 2009;13:1-134.

2 Parkes J, Roderick P, Harris S, et al. Enhanced liver fibrosis test can predict clinical outcomes in patients with chronic liver disease. Gut 2010;59:1245-51.

3 Pratt DS, Kaplan MM. Evaluation of abnormal liverenzyme results in asymptomatic patients. N Engl J Med 2000;342:1266-71.

4 Tapper EB, Saini SD, Sengupta N. Extensive testing or focused testing of patients with elevated liver enzymes. J Hepatol 2017;66:313-9.

5 Bellentani S. The epidemiology of non-alcoholic fatty liver disease. Liver Int 2017;37(Suppl 1):81-4.

6 Masarone M, Rosato V, Dallio M, et al. Epidemiology and Natural History of Alcoholic Liver Disease. Rev Recent Clin Trials 2016;11:167-74.

7 Welzel TM, Bhardwaj N, Hedskog C, et al. Global epidemiology of HCV subtypes and resistance-associated substitutions evaluated by sequencing-based subtype analyses. J Hepatol 2017;67:224-36.

8 Bouttell J, Lewsey J, Geue C, et al. The SCottish Alcoholic Liver disease Evaluation: A Population-Level Matched Cohort Study of Hospital-Based Costs, 1991-2011. PLoS One 2016;11:e0162980.

9 Rice P. Encouraging results from the Scottish alcohol brief intervention programme. BMJ 2017;356:j1376.

10 Lilford RJ, Bentham L, Girling A, et al. Birmingham and Lambeth Liver Evaluation Testing Strategies (BALLETS): a prospective cohort study. Health Technol Assess 2013;17:1-307.

11 Radcke S, Dillon JF, Murray AL. A systematic review of the prevalence of mildly abnormal liver function tests and associated health outcomes. Eur J Gastroenterol Hepatol 2015;27:1-7.

12 Dalkey N, Helmer O. An Experimental Application of the DELPHI Method to the Use of Experts. Manage Sci 1963;9:458-67.

13 Angulo P, Bugianesi E, Bjornsson ES, et al. Simple noninvasive systems predict long-term outcomes of patients with nonalcoholic fatty liver disease. Gastroenterology 2013;145:782-9.

14 Sterling RK, Lissen E, Clumeck N, et al. Development of a simple noninvasive index to predict significant fibrosis in patients with $\mathrm{HIV} / \mathrm{HCV}$ coinfection. Hepatology 2006;43:1317-25.

15 Wai CT, Greenson JK, Fontana RJ, et al. A simple noninvasive index can predict both significant fibrosis and cirrhosis in patients with chronic hepatitis C. Hepatology 2003;38:518-26.

16 Harris R, Harman DJ, Card TR, et al. Prevalence of clinically significant liver disease within the general population, as defined by non-invasive markers of liver fibrosis: a systematic review. Lancet Gastroenterol Hepatol 2017;2:288-97. 